\title{
Effects of low-dose flosequinan on left ventricular systolic and diastolic chamber performance
}

Flosequinan (manoplax) is a new vasodilating agent for the treatment of congestive heart failure. Although it may have several mechanisms of action, whether it has effects on left ventricular inotropic or luisotropic events in hemodynamically relevant low doses when added to standard therapy for congestive heart failure is unknown. Ten patients with dilated congestive cardiomyopathy who were receiving standard therapy for heart failure were studied. A bipolar right atrial pacing catheter was used to maintain a constant heart rate. A TF thermodilution catheter was used to measure right heart pressures and obtain cardiac outputs. An $8 F$ micromanometer catheter was used to measure left ventricular and ascending aortic pressures. Gated equilibrium radionuclide angiography was performed both before and during a steady-state infusion of flosequinan. The average flosequinan infusion rate was $2.03 \pm 0.85$ $\mathrm{mg} / \mathrm{min}$, and the total administered dose averaged $84 \pm 35 \mathrm{mg}$. The hemodynamic data documented substantial systemic vasodilation manifest by a reduction in right atrial pressure $(p=0.01)$, mean pulmonary artery pressure $(p<0.0001)$, pulmonary capillary wedge pressure $(p<0.0001)$, and left ventricular end-diastolic pressure $(p<0.0001)$. These hemodynamic changes were associated with increases in cardiac index $(p=0.01)$ and left ventricular ejection fraction $(p=0.02)$ and reductions in mean aortic pressure $(p=0.02)$, systemic vascular resistance $(p=0.01)$, and left ventricular volumes $(p<0.05)$. There was, however, no significant effect on left ventricular contractile function measured by end-systolic pressure-volume relationship $\left(E_{\max }\right), E_{\max }$ corrected for the change in left ventricular volume, or preload recruitable stroke work $\left(M_{s w}\right)$. In contrast, there was an improvement in isovolumic relaxation manifest by an increase in maximum rate of fall of left ventricular pressure standardized for left ventricular end-systolic pressure $\left.\left[(-) \mathrm{dP}_{\mathrm{dt}} \mathrm{dt}_{\mathrm{min}} / \mathrm{P}_{\mathrm{es}}\right] ; \boldsymbol{p}=0.02\right)$, an acceleration in the rate of isovolumic relaxation $(p=0.01)$, and an improvement in left ventricular chamber stiffness $(p=0.02)$. These data indicate that when flosequinan, a new therapeutic agent for the treatment of congestive heart failure, is administered in hemodynamically relevant low doses to patients with dilated congestive cardiomyopathy who were receiving standard therapy for heart failure, left ventricular pump function and diastolic function is further improved. There was, however, no signiflcant effect on left ventricular contractility. This study emphasizes that new therapeutic agents like flosequinan, when administered in lower doses to avoid the potential deleterious effects of enhanced inotropy, may be useful additions to standard therapy in patients with congestive heart failure. (AM HEART J 1994;128:124-33.)

\section{Mark R. Starling, MD Ann Arbor, Mich.}

Flosequinan (manoplax) is a newly developed, orally active, 7-fluorinated quinoline that has unique vasodilator properties on both the systemic venous and arterial circulations. ${ }^{1}$ Several mechanisms for this vasodilating action of flosequinan have been pro-

From the Division of Cardiology, Department of Internal Medicine, The University of Michigan and Veterans Administration Medical Centers.

Supported by Boots Pharmaceuticals, Inc. (USA) and the Veterans Administration, Washington, D. C.

Received for publication Sept. 14, 1993; accepted Nov. 3, 1993.

Reprint requests: Mark R. Stariing, MD, Department of Internal Medicine, Division of Cardiology, VA Medical Center, 2215 Fuller Road, Ann Arbor, MI 48105 .

Copyright ${ }^{(0)} 1994$ by Mosby-Year Book, Inc.

$0002-8703 / 94 / \$ 3.00+0 \quad \mathbf{4} / \mathbf{1} / \mathbf{5 4 5 4 3}$ posed. ${ }^{2}$ Some investigations have also suggested that this agent may have weak inotropic potential resulting from nonspecific phosphodiesterase inhibition. ${ }^{2}$ Clinical studies in which flosequinan was administered to patients with congestive heart failure have demonstrated hemodynamic improvement consistent with the predominant systemic venous and arterial vasodilating properties of this therapeutic agent. ${ }^{4}$ These hemodynamic improvements appear to also be associated with improvements in clinical symptoms and exercise tolerance. ${ }^{5}$ Although it is assumed that the major beneficial hemodynamic effects of flosequinan in patients with congestive heart failure are related to a vasodilating action, they may also be the result of weak inotropic or luisotropic ac- 
tions. There is evidence in human beings that this weak inotropic effect may be evident at higher doses of flosequinan, ${ }^{6}$ but the benefits of flosequinan to patients with congestive heart failure may be predominantly related to the additive vasodilating potential of the agent rather than the inotropic effects observed at higher doses, which may be detrimental in some patients with congestive heart failure. Accordingly, this investigation was undertaken to determine whether hemodynamically relevant low doses of flosequinan have beneficial effects on left ventricular systolic and diastolic chamber performance in addition to their known systemic venous and arterial vasodilating action in a select group of patients with dilated congestive cardiomyopathy, who were receiving standard therapy for heart failure.

\section{METHODS}

Patients. The patient population consisted of 10 men with dilated congestive cardiomyopathy who ranged in age from 42 to 67 years (mean $53 \pm 10$ years). All patients had normal sinus rhythm, had never experienced an ischemic myocardial event, had a gated equilibrium radionuclide angiogram demonstrating a left ventricular ejection fraction of $\leq 0.40$, and had normal coronary anatomy at cardiac catheterization. All patients had been receiving a stable therapeutic regimen of digitalis, a diuretic, and an angiotensin-converting enzyme inhibitor for at least 2 months. No patients were in New York Heart Association clinical class I, 2 were in class II, 8 were in class III, and none were in class IV. Each patient provided written informed consent for this protocol on forms approved by either the Institutional Review Board at the University of Michigan Medical Center or the Human Studies Committee at the Veterans Affairs Medical Center in Ann Arbor, Mich.

Protocol. After diagnostic right and left heart catheterizations, each patient entered the protocol. A bipolar pacing catheter was placed in the right atrial appendage to maintain a constant heart rate. A 7F thermodilution catheter was used to perform a right heart catheterization, and it remained in the pulmonary artery throughout the protocol. Both the proximal and distal ports were connected to fluid transducers to measure pulmonary artery, pulmonary capillary wedge, and right atrial pressures. An $8 \mathrm{~F}$ micromanometer catheter (SPC-784A, Millar Instruments, Inc., Houston, Texas) was calibrated and positioned in the left ventricle to measure both left ventricular and ascending aortic pressures. In vivo red blood cell labeling with 30 to $35 \mathrm{mCi}$ of technetium $99 \mathrm{~m}$ was achieved for gated equilibrium radionuclide angiography. A gamma scintillation camera was brought into the cardiac catheterization laboratory and positioned to optimally separate the right and left ventricles in the plane of the interventricular septum.

Each patient was allowed to equilibrate; right and left heart pressures were recorded, and thermodilution cardiac outputs were obtained in triplicate. Micromanometer left ventricular and aortic pressures and gated equilibrium radionuclide angiograms were acquired under control condi- tions and during methoxamine or nitroprusside infusions to obtain two additional steady-state left ventricular loading conditions.

After completing this phase of the protocol, each patient was allowed to return to baseline, which was defined as pressure within $10 \%$ of the original baseline hemodynamic pressures. All pressures and cardiac outputs were repeated. A steady-state intravenous infusion of flosequinan was begun and incrementally increased to achieve a $5 \mathrm{~mm} \mathrm{Hg} \mathrm{de-}$ crease in pulmonary capillary wedge pressure or a $20 \mathrm{~mm}$ $\mathrm{Hg}$ decrease in left ventricular systolic pressure. Once one of these hemodynamic end points was reached, the right and left heart pressures and cardiac outputs were repeated, blood samples were drawn for drug levels, and the micromanometer pressures and radionuclide angiograms were again acquired under basal conditions and during methoxamine or nitroprusside infusions to obtain two additional steady-state left ventricular loading conditions. The average flosequinan infusion rate was $2.03 \pm 0.85 \mathrm{mg} / \mathrm{min}$ with a range of 0.67 to $3.5 \mathrm{mg} / \mathrm{min}$ for a total administered dose of $84 \pm 35 \mathrm{mg}$ (range 53 to $125 \mathrm{mg}$ ).

After this phase of the protocol was completed, the flosequinan infusion was discontinued. Right and left heart pressures and cardiac outputs were repeated 15 minutes after completion of the flosequinan infusion, and a final blood sample was drawn for drug levels. All 10 patients completed the protocol without complications.

Hemodynamics. At each point during the protocol, phasic and mean right atrial, pulmonary artery, and pulmonary capillary wedge pressures were recorded. Cardiac outputs were performed in triplicate with the thermodilution technique. Micromanometer left ventricular and aortic pressures were recorded at $100 \mathrm{~mm} / \mathrm{sec}$ paper speed. The micromanometer left ventricular pressures were digitized as previously described from this laborato$\mathrm{ry}^{7}$ and interpolated to provide corresponding left ventricular pressures for each radionuclide left ventricular volume determination.

Radionuclide angiography. Gated equilibrium radionuclide angiograms were acquired and processed as previously described from this laboratory. ${ }^{8,9}$ Each radionuclide angiogram was acquired into $30 \mathrm{msec}$ frames for 250 cardiac cycles. During the midportion of each radionuclide acquisition a $2 \mathrm{ml}$ blood sample was drawn and the time recorded. Blood samples were later counted for $2 \mathrm{~min}$ and the time delay recorded for decay correction. At completion of the study, distance measurements were made for each patient to determine the distance from the left ventricle to the gamma scintillation camera for attenuation correction. Left ventricular volumes were calculated on a frame-byframe basis with background-subtracted hand-drawn region-of-interest left ventricular count data, decay-corrected blood sample counts, and attenuation correction. ${ }^{8}$

Data analysis. Cardiac index was calculated by dividing cardiac output by body surface area. Stroke volume index was calculated by dividing $\mathrm{CI}$ by the corresponding heart rate. Mean aortic pressure was obtained by adding one third of the aortic pulse pressure to the aortic diastolic pressure. Left ventricular stroke work index was obtained 


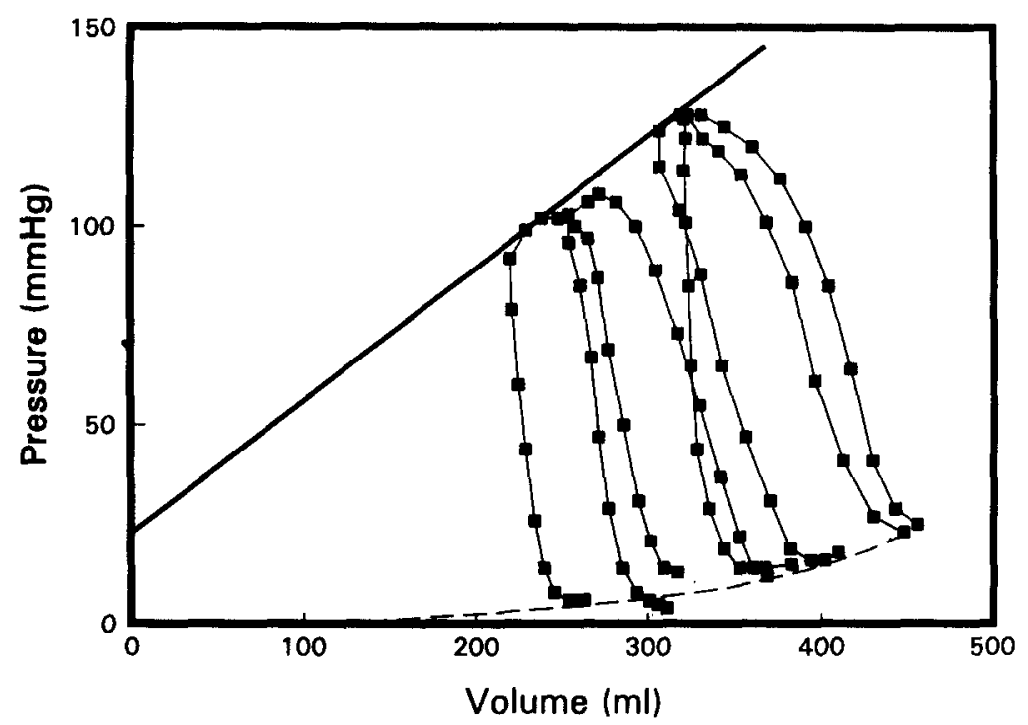

Fig. 1. Calculation of left ventricular chamber elastance and left ventricular diastolic chamber constant are shown for representative patient with dilated congestive cardiomyopathy. Micromanometer left ventricular pressures are plotted on ordinant and radionuclide left ventricular volumes are plotted on abscissa. Multiple pressure-volume loops are generated and maximum left ventricular chamber elastance $\left(E_{\max }\right)$, a relatively load-independent index of left ventricular contractility (solid line), and left ventricular diastolic chamber compliance ( $k$, dashed line) were calculated as shown from these data.

by multiplying mean aortic pressure by stroke volume index; the result was multiplied by 0.0136 to convert from millimeters of mercury per milliliter per square meter to gram-meters per square meter. Systemic vascular resistance was calculated by multiplying the difference between mean aortic and right atrial pressure by cardiac outpul and multiplying the result by 80 dynes $\cdot \mathrm{sec} \cdot \mathrm{cm}^{-5}$.

I eft ventricular systolic function was assessed in several ways. We evaluated the isovolumic phase of systole by calculating the maximum rate of increase of left ventricular pressure $\left.\left[(+) \mathrm{dP} / \mathrm{dt}_{\max }\right]\right) .{ }^{7}$ We also assessed several other ejection phase and contractile indexes. Left ventricular ejection fraction was calculated in the standard fashion by using the radionuclide left ventricular end-diastolic and end-systolic volumes. Corresponding micromanometer left ventricular pressures and radionuclide left ventricular volumes were plotted to generate multiple pressure-volume loops (Fig. 1). The pressure-volume loops from the basal condition and during the steady-state flosequinan infusion were subjected to calibrated planimetry. These areas were multiplied by 0.0136 to convert from millimeters of mercury per milliliter to gram-meters. Because left ventricular stroke work is preload dependent, ${ }^{10}$ we plotted the left ventricular stroke work values from each pressure-volume loop against their corresponding left ventricular end-diastolic volumes to obtain a slope reflective of preload recruitable stroke work $\left(\mathrm{M}_{\mathrm{sw}}, \mathrm{Fig} .2\right)$, an index of left ventricular contractility. We also used left ventricular chamber elastance, another relatively load-independent index of left ventricular contractility. ${ }^{11-14}$ By using the multiple pressure-volume loops generated under the basal condition and during the steady-state flosequinan infusion, isoch- ronal pressure-volume points were subjected to linear re gression analyses to obtain a maximum slope, $\mathrm{E}_{\max }$ (Fig. 1) Because $E_{\max }$ can be affected by left ventricular size, ${ }^{15-17} \mathrm{w}$ subjected the $\mathrm{E}_{\max }$ values to a mathematical correction to adjust for the contribution of changes in heart size on $\mathrm{E}_{\max }$

Left ventricular diastolic function was assessed in severa ways. Left ventricular isovolumic relaxation was evaluatec by using left ventricular maximum rate of fall of left ven tricular pressure $\left[(-) \mathrm{dP} / \mathrm{dt}_{\min }\right]$. Because this index of iso volumic relaxation is pressure dependent, ${ }^{18}(-) \mathrm{dP} / \mathrm{dt}_{\mathrm{mi}}$ was standardized to the micromanometer left ventricula] pressure $\left(\mathrm{P}_{\mathrm{es}}\right)$ at $(-) \mathrm{dP} / \mathrm{dt}_{\min }\left[(-) \mathrm{dP} / \mathrm{dt}_{\mathrm{min}} / \mathrm{P}_{\mathrm{es}}\right]$. We alsc quantitated the effects of flosequinan on the rate $\mathrm{o}$ : isovolumic pressure decline over the time from $(-) \mathrm{dP} / \mathrm{dt}$. $\min$ to $5 \mathrm{~mm} \mathrm{Hg}$ above left ventricular end-diastolic pressure of the next beat using the method of Weiss et al., ${ }^{19,}{ }^{20}$ which is given by the equation $P(t)=P_{0} e^{-t / T}$. The natural loga rithmic transformation of this equation yields $\ln P=-\mathrm{lt}$, $\mathrm{T}+\ln \mathrm{P}_{0}$, where the time constant (Tau) is the negative reciprocal of the slope $(-1 / \mathrm{T})$. Finally, we evaluated left ventricular chamber stiffness. ${ }^{21}$ This index was obtained by using nonlinear regression of the corresponding micromanometer left ventricular end-diastolic pressures and radionuclide left ventricular end-diastolic volumes to obtain a chamber stiffness constant (Fig. 1).

Pharmacologic assay. Flosequinan and BTS 53,554, the active metabolite of flosequinan, were assayed from plasma samples by the method of Slegowski et al.,22 which was modified to use two overlapping standard curves to diminish variance at the lower range of detection. The high range was $0,200,500,2000,3333$, and $5000 \mathrm{ng} / \mathrm{ml}$, and the low range was $0,50,100,200$, and $500 \mathrm{ng} / \mathrm{ml}$ of either flose- 


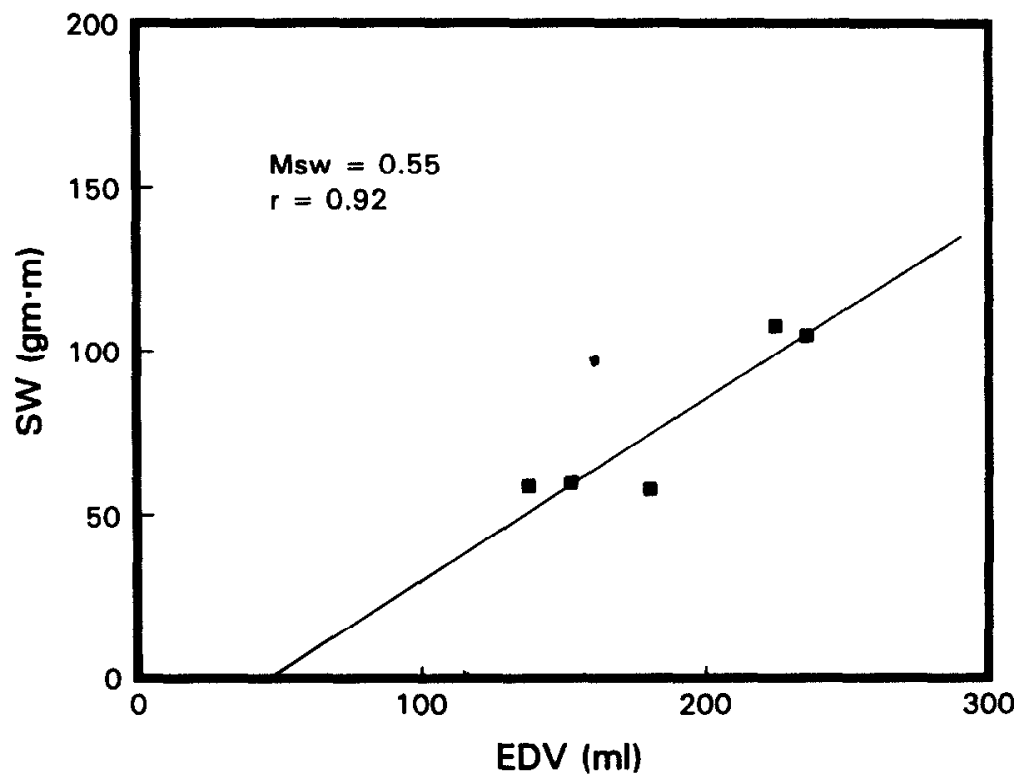

Fig. 2. Representative example of calculation of preload recruitable stroke work is shown. Left ventricular stroke work $(S W)$ values are plotted on ordinate and left ventricular end-diastolic volumes $(E D V)$ are plotted on abscissa over full range of loading conditions. Slope reflects preload recruitable stroke work $\left(M_{s w}\right)$, a relatively load-independent index of left ventricular contractility.

Table I. Hemodynamics $(n=10)$

\begin{tabular}{|c|c|c|c|}
\hline & Baseline & $\begin{array}{c}\text { With } \\
\text { finsequinan }\end{array}$ & $P$ Value \\
\hline HR (beats/min) & $87 \pm 10$ & $88 \pm 9$ & 0.37 \\
\hline $\mathrm{RAP}$ (mm Hg) & $9 \pm 2$ & $8 \pm 2$ & 0.01 \\
\hline MPAP (mm Hg) & $31 \pm 9$ & $23 \pm 10$ & $<0.0001$ \\
\hline PCWP (mm Hg) & $21 \pm 10$ & $12 \pm 9$ & $<0.0001$ \\
\hline $\mathrm{CI}\left(\mathrm{L} / \mathrm{min} / \mathrm{m}^{2}\right)$ & $2.61 \pm 0.78$ & $2.82 \pm 0.67$ & 0.01 \\
\hline SVI $\left(\mathrm{ml} / \mathrm{m}^{2}\right)$ & $30 \pm 9$ & $32 \pm 8$ & 0.04 \\
\hline LVSP (mm Hg) & $122 \pm 25$ & $115 \pm 21$ & 0.06 \\
\hline LVEDP (mm Hg) & $28 \pm 5$ & $16 \pm 8$ & $<0.0001$ \\
\hline $\mathrm{AoP}(\mathrm{mm} \mathrm{Hg})$ & $91 \pm 12$ & $84 \pm 11$ & 0.02 \\
\hline SWI $\left(\mathrm{g}-\mathrm{m} / \mathrm{m}^{2}\right)$ & $30 \pm 12$ & $32 \pm 11$ & 0.13 \\
\hline SVR (dynes $\cdot \mathrm{sec} \cdot \mathrm{cm}^{-5}$ ) & $1328 \pm 528$ & $1108 \pm 341$ & 0.01 \\
\hline PVR (dynes $\cdot \mathrm{sec} \cdot \mathrm{cm}^{-5}$ ) & $167 \pm 123$ & $155+73$ & 0.58 \\
\hline
\end{tabular}

$H R$, heart rate; $R A P$, right atrial pressure; $M P A P$, mean pulmonary artery pressure; $P C W P$, pulmonary capillary wedge pressure; $C I$, cardiac index; $S V I$, stroke volume index; $L V S P$, left ventricular systolic pressure; $L V E D P$, left ventricular end-diastolic pressure; $A o P$, mean aortic pressure; $S W I$, stroke work index; $S V R$, systemic vascular resistance; $P V R$, pulmonary vascular resistance.

quinan or BTS 53,554 . The standard curves for both ranges of flosequinan were linear $(r=0.999)$, with a slope of 1841 or $1842 \mathrm{ng} / \mathrm{ml}$, a coefficiency of variation of $1.5 \%$, and a mean SEE of $32.7 \mathrm{ng} / \mathrm{ml}$ equivalents for the high range and $4.8 \mathrm{ng} / \mathrm{ml}$ equivalents for the low range. The interbatch sample recovery for $50 \mathrm{ng} / \mathrm{ml}$ samples was $108.7 \%$ with a coefficiency of variation of $7.9 \%$. Intrabatch recovery and coefficiency of variation were $100.6 \%$ and $11.8 \%$, respectively. All values for BTS 53,554 were comparable; thus the assay had acceptable precision and accuracy down to 50 $\mathrm{ng} / \mathrm{ml}$ for both flosequinan and its metabolite.
Statistical analysis. All data are presented as the mean $\pm \mathrm{SD}$. Continuous variables were compared by paired $t$ tests. Differences were statistically significant when a probability value of $\leq 0.05$ was obtained.

\section{RESULTS}

Hemodynamic data. The hemodynamic data during the baseline condition and the steady-state flosequinan infusion are shown in 'Table 1 . Heart rate was unchanged as a result of right atrial appendage pacing. The mean right atrial pressure fell from $9 \pm 2$ to 
$\operatorname{Emax}(\mathrm{mmHg} / \mathrm{ml})$

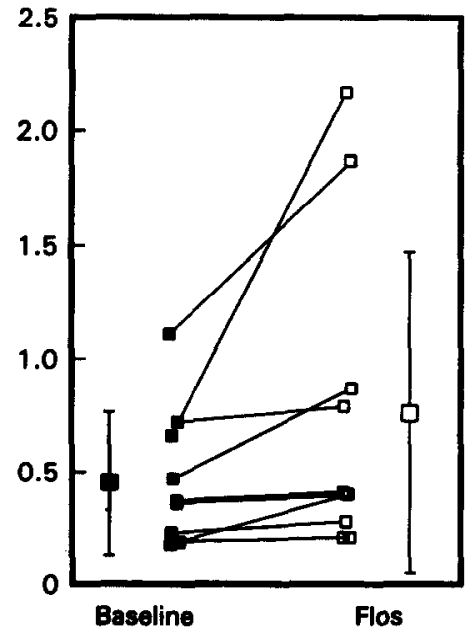

Corr Emax $(\mathrm{mmHg} / \mathrm{ml})$

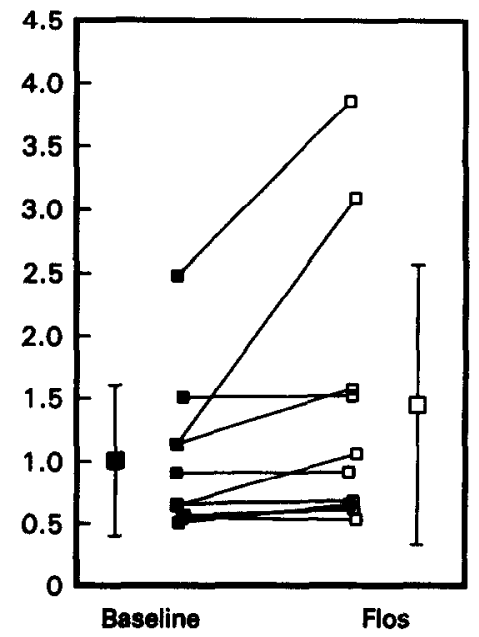

Fig. 3. Left, Individual and mean left ventricular chamber elastance $\left(E_{\max }\right)$ values at baseline and during steady-state flosequinan (Flos) infusion are shown. Right, Individual and mean $\mathrm{E}_{\max }$ values after correction for changes in left ventricular end-diastolic volume are also shown. Differences in individual responses are evident, and average $\mathbf{E}_{\max }$ and $\mathrm{E}_{\max }$ corrected for end-diastolic volume (Corr $E_{\max }$ ) were not affected.

Table II. Left ventricular chamber performance and ventriculo-arterial coupling relations

\begin{tabular}{|c|c|c|c|}
\hline & Baseline & $\begin{array}{c}\text { With } \\
\text { flosequinan }\end{array}$ & $P$ Value \\
\hline$(+) \mathrm{dP} / \mathrm{dt}_{\max }(\mathrm{mm} \mathrm{Hg} / \mathrm{sec})$ & $1143 \pm 382$ & $1201 \pm 624$ & 0.65 \\
\hline EDV $(\mathrm{ml})$ & $461 \pm 210$ & $382 \pm 174$ & 0.03 \\
\hline $\mathrm{ESV}(\mathrm{ml})$ & $345 \pm 173$ & $268 \pm 142$ & 0.02 \\
\hline EF & $0.27 \pm 0.07$ & $0.32 \pm 0.10$ & 0.02 \\
\hline $\mathrm{E}_{\max }(\mathrm{mm} \mathrm{Hg} / \mathrm{ml})$ & $0.45 \pm 0.30$ & $0.76 \pm 0.70$ & 0.07 \\
\hline $\mathrm{E}_{\max } \operatorname{CORR}(\mathrm{mm} \mathrm{Hg} / \mathrm{ml})$ & $1.01 \pm 0.61$ & $1.45 \pm 1.14$ & 0.06 \\
\hline $\mathrm{M}_{\mathrm{sw}}(\mathrm{gm}-\mathrm{m} / \mathrm{ml})$ & $0.24 \pm 0.15$ & $0.38 \pm 0.26$ & 0.08 \\
\hline$(-) \mathrm{dP} / \mathrm{dt}_{\min }(\mathrm{mm} \mathrm{Hg} / \mathrm{sec})$ & $1060 \pm 244$ & $1283 \pm 510$ & 0.09 \\
\hline$(-) \mathrm{dP} / \mathrm{dt}_{\min } / \mathrm{P}_{\mathrm{es}}\left(\mathrm{sec}^{-1}\right)$ & $15 \pm 4$ & $19 \pm 6$ & 0.02 \\
\hline Tau (msec) & $67 \pm 17$ & $53 \pm 13$ & 0.01 \\
\hline $\mathrm{k}(\mathrm{mm} \mathrm{Hg} / \mathrm{ml})$ & $0.07 \pm 0.04$ & $0.05 \pm 0.05$ & 0.02 \\
\hline
\end{tabular}

$(+) d P / d t_{\max }$, maximum rate of rise of left ventricular pressure; $E D V$, end-diastolic volume; $E S V$, end-systolic volume; $E F$, ejection fraction; $M_{s w}$, preloac recruitable stroke work; $E_{\max }$, end-systolic pressure-volume relationship; $E_{\max } C O R R$, end-systolic pressure-volume relationship corrected for volume $(-) d P / d t_{\min }$, maximum rate of fall of left ventricular pressure; $(-) d P / d t_{\min } / P_{e s}$, maximum rate of fall of left ventricular pressure standardized for left ven tricular end-systolic pressure; Tau, isovolumic relaxation rate; $k$, left ventricular diastolic chamber stiffness.

$8 \pm 2 \mathrm{~mm} \mathrm{Hg}(p=0.01)$. The mean pulmonary artery pressure fell from $31 \pm 9$ to $23 \pm 10 \mathrm{~mm} \mathrm{Hg}$ ( $p<$ 0.0001 ), and the mean pulmonary capillary wedge pressure was reduced from $21 \pm 10$ to $12 \pm 9 \mathrm{~mm} \mathrm{Hg}$ $(p<0.0001)$. The cardiac index increased $\left(2.61 \pm 0.78\right.$ to $\left.2.82 \pm 0.67 \mathrm{~L} / \mathrm{min} / \mathrm{m}^{2}, p=0.01\right)$, as did the stroke volume index $\left(30 \pm 9\right.$ to $32 \pm 8 \mathrm{ml} / \mathrm{m}^{2}$, $p=0.04$ ). Left ventricular systolic pressure was marginally affected $(122 \pm 25$ to $115 \pm 21 \mathrm{~mm} \mathrm{Hg}$, $p=0.06$ ), and left ventricular end-diastolic pressure was reduced from $28 \pm 5$ to $16 \pm 8 \mathrm{~mm} \mathrm{Hg}$ $(p<0.0001)$. Despite a marginal effect on left ven- tricular systolic pressure, mean AoP decreased from $91 \pm 12$ to $84 \pm 11 \mathrm{~mm} \mathrm{Hg}(p=0.02)$. Consequently although left ventricular stroke work was unaffected systemic vascular resistance decreased from $1328 \pm 528$ to $1108 \pm 341$ dynes $\cdot$ sec $\cdot \mathrm{cm}^{-5}$ ( $p=$ 0.01 ), and pulmonary vascular resistance was un. changed.

Left ventricular systolic function. The effects of flosequinan on left ventricular systolic function are shown in Table II. The left ventricular $(+) \mathrm{dP} / \mathrm{dt}_{\max }$ was not affected by the flosequinan infusion. Left ventricular end-diastolic and end-systolic volumes 
were reduced ( $p=0.03$ and $p=0.02$, respectively), which lead to an increase in left ventricular ejection fraction from $0.27 \pm 0.07$ to $0.32 \pm 0.10(p=0.02)$. However, left ventricular SWI did not increase with the flosequinan infusion.

When the relatively load-independent, end-systolic index of left ventricular contractility, $\mathrm{E}_{\max }$, was assessed, no significant improvement was noted. The individual and mean values for $\mathrm{E}_{\max }$ are shown in Fig. 3 . The average value increased from $0.45 \pm 0.30$ to $0.76 \pm 0.70 \mathrm{~mm} \mathrm{Hg} / \mathrm{ml}(p=0.07)$. The data in Fig. 3 show that these mean values were substantially affected by two patients who had marked increases in left ventricular chamber elastance, whereas 8 of the 10 patients had little or no change in $\mathrm{E}_{\max }$. Because $\mathrm{E}_{\max }$ may have been affected by the changes in left ventricular size induced by flosequinan, the individual $\mathrm{E}_{\max }$ values were adjusted for the effects of the changes in heart size, and they also showed a marginal but insignificant change $(1.01 \pm 0.61$ to $1.45 \pm$ $1.14 \mathrm{~mm} \mathrm{Hg} / \mathrm{ml}, p=0.06$ ). Because $\mathrm{E}_{\max }$ can be affected by heart size and can be nonlinear in these low contractile ranges, preload recruitable SW was also used to assess left ventricular contractility. As shown in Table II, this contractile index was also not significantly affected by flosequinan in the doses used in this investigation $(0.24 \pm 0.15$ vs $0.38 \pm 0.26 \mathrm{gm}$ $\mathrm{m} / \mathrm{ml}, p=0.08$ ).

Left ventricular diastolic function. The effects of flosequinan on left ventricular diastolic function are also shown in Table II. Left ventricular (-)dP/dt $t_{\min }$ was not affected by the flosequinan infusion. However, because left ventricular pressure was reduced, when $(-) \mathrm{dP} / \mathrm{dt}_{\min }$ was standardized to $\mathrm{P}_{\mathrm{es}}$ at $(-) \mathrm{dP} /$ $\mathrm{dt}_{\min }$, the isovolumic pressure decay significantly accelerated from $15 \pm 4$ to $19 \pm 6 \sec ^{-1}(p=0.02)$. This was confirmed by an acceleration in Tau from $67 \pm 17$ to $53 \pm 13 \mathrm{msec}(p=0.01$, Fig. 4 ). All patients demonstrated a systematic acceleration in the rate of isovolumic pressure decline. Left ventricular chamber stiffness was also improved by the flosequinan infusion. The left ventricular chamber stiffness constant $(k)$ was reduced from $0.07 \pm 0.04$ to $0.05 \pm 0.05 \mathrm{~mm} \mathrm{Hg} / \mathrm{ml}(p=0.02$, Fig. 5). With one exception, all patients studied demonstrated a systematic reduction in left ventricular chamber stiffness, as is illustrated for a representative patient in Fig. 5.

Pharmacologic factors. Flosequinan and single major active metabolite BTS 53,554 plasma levels substantially varied in these patients with congestive heart failure. The peak flosequinan plasma level ranged from 352 to $4554 \mathrm{ng} / \mathrm{ml}(1974 \pm 1384 \mathrm{ng} / \mathrm{ml})$,

\section{Tau (msec)}

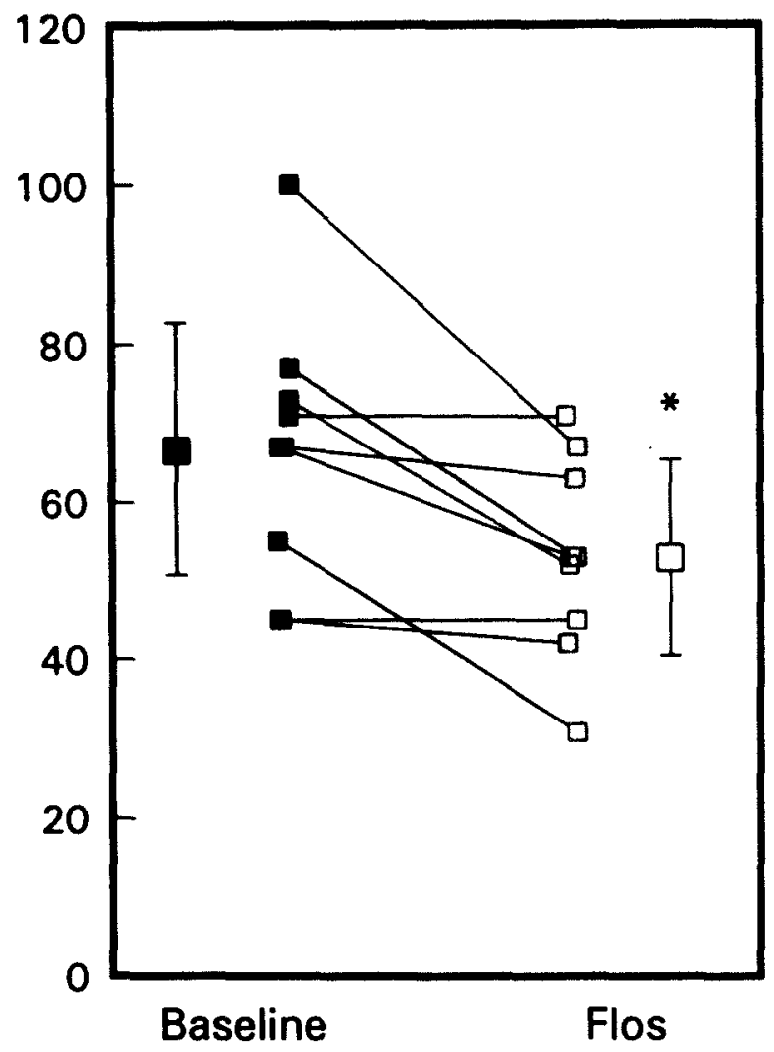

Fig. 4. Left, Individual and mean Tau values at baseline and during steady-state flosequinan (Flos) are shown. Systematic reductions in individual values are evident.

and the BTS 53,554 plasma level varied between 0 and $552 \mathrm{ng} / \mathrm{ml}(426 \pm 232 \mathrm{ng} / \mathrm{ml})$.

\section{DISCUSSION}

Flosequinan is a new therapeutic agent for the treatment of congestive heart failure, which has mixed systemic venous and arterial vasodilating action. Although the specific mechanisms of action of flosequinan are unclear, it is apparent that the mechanisms by which flosequinan exerts these beneficial hemodynamic effects differ from those of therapeutic agents currently in use for the treatment of congestive heart failure. Data from rat aortic smooth-muscle preparations demonstrate that the concentration of flosequinan required to block $50 \%$ of a norepinephrine-induced contraction is substantially less than that needed to produce a comparable reduction in a potassium chloride-induced contraction. ${ }^{23}$ These data suggest that flosequinan may have a greater effect on modification of intracellular calcium handling than on transmembrane calcium transients. 
LV Chamber

Compliance $(\mathrm{mmHg} / \mathrm{ml})$

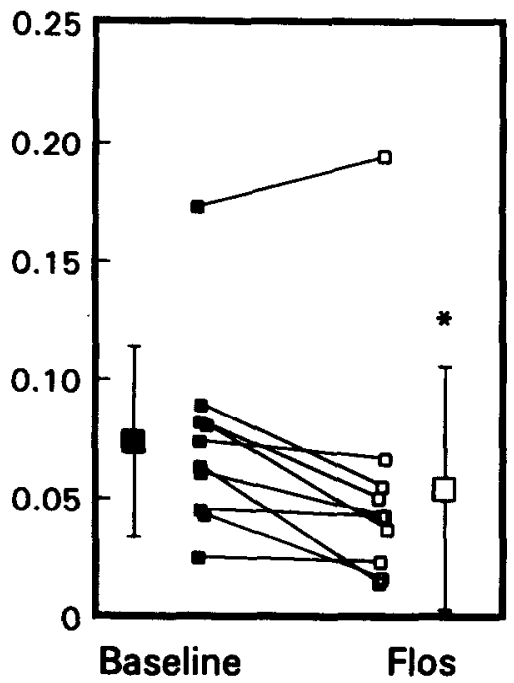

\section{LVEDP $(\mathrm{mmHg})$}

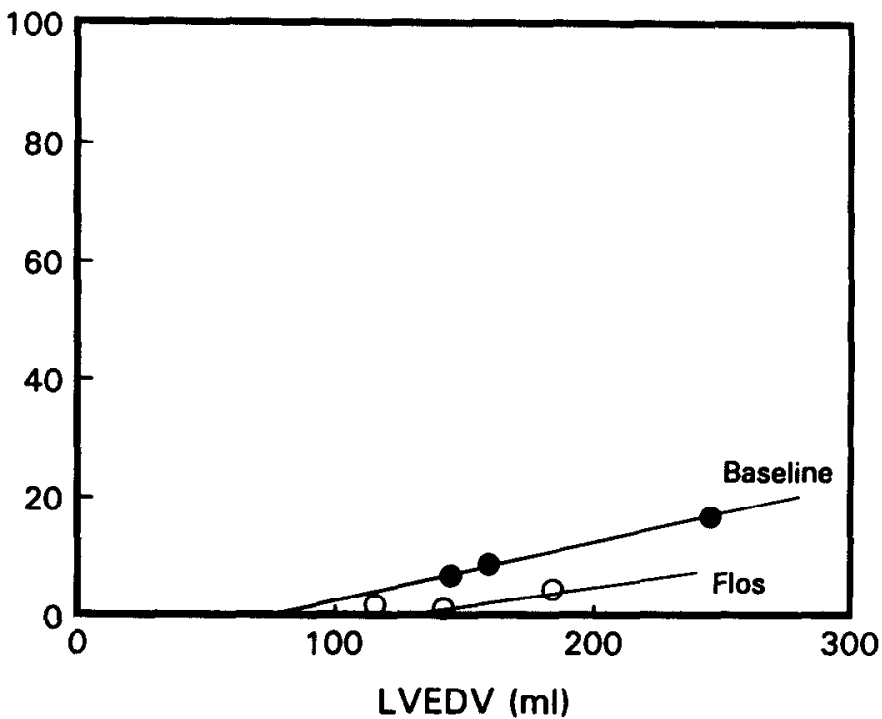

Fig. 5. Left, Individual and mean left ventricular $(L V)$ chamber compliance values are shown in similar format. Systematic reductions in individual left ventricular chamber compliance constant are evident. Significant difference is noted. Right, Representative example from patient with dilated congestive cardiomyopathy of left ventricular diastolic chamber stiffness is shown under baseline conditions and during steady-state flosequinan (Flos) infusion. Downward displacement with flattening of chamber compliance curve is evident. $L V E D P$, Left ventricular end-diastolic pressure; $L V E D V$, left ventricular end-diastolic volume.

Further studies from the rat aortic preparation demonstrate that flosequinan can relax contractions induced by phorbol dibutyrate. ${ }^{24}$ Because phorbol dibutyrate-induced contractions occur through the stimulation of protein kinase $C$, it has been suggested that flosequinan may prevent the stimulation of protein kinase $\mathrm{C}$ as an alternative mode of action. However, the concentrations of flosequinan required to produce $50 \%$ relaxation of a phorbol dibutyrate contraction are greater than those needed to produce a similar block of a norepinephrine contraction in the same preparation, suggesting that this mechanism may also be less important than the effects of flosequinan on intracellular calcium handling.

More recent studies performed with the rat aortic preparation demonstrate that flosequinan prevents the production of inositol 1,4,5-triphosphate and to a lesser extent protein kinase $\mathrm{C}$ activation produced by endothelin-1 effector-receptor coupling. ${ }^{25}$ These data coupled with prior data ${ }^{23,24,26}$ suggest that flosequinan may block the effector-receptor activation of $\mathrm{G}$ transduction proteins which, in the presence of guanosine triphosphate, stimulate phospholipase C. Activation of membrane-bound phospholipase $\mathrm{C}$ catalyzes the hydrolysis of phosphoinositides to the secondary messengers inositol $1,4,5$-triphosphate and diacylglycerol. Inositol 1,4,5-triphosphate increases intracellular calcium levels by releasing calcium from intracellular stores; diacylglycerol does so by stimulating protein kinase $\mathrm{C}$ to increase transmembrane calcium transients. Thus it can be postulated that flosequinan may act in vascular smooth muscle by blocking the production of secondary messengers, either at the transduction protein level or by the activation of phospholipase C.

These mechanisms of action may explain the systemic venous and arterial vasodilating action observed with flosequinan; however, there are additional possible mechanisms of action for flosequinan on myocardial tissue. ${ }^{3,27}$ In guinea pig left ventricular myocardium, flosequinan in high concentrations has caused an increase in cyclic adenosine monophosphate and guanosine monophosphate. These data suggest that flosequinan may have an effect on phosphodiesterase activity. Although this effect was noted only at high concentrations of flosequinan, it does suggest that flosequinan may have a positive inotropic effect on myocardial tissue. There have been several studies in human beings that have resulted in conflicting conclusions regarding the inotropic po- 
tential of flosequinan. ${ }^{6,28,29}$ Initially, Haas et al. ${ }^{28}$ studied 10 patients with congestive heart failure before and after the administration of $100 \mathrm{mg}$ of flosequinan. They observed favorable alterations in loading conditions that improved left ventricular pump function, but inotropic parameters did not change significantly compared to placebo. Subsequently, Corin et al. ${ }^{29}$ studied a similar group of 10 patients with congestive heart failure and noted that there was a modest but significant increase in $\mathrm{dP} / \mathrm{dt}_{\max }$ of approximately $10 \%$. More recently, Burstein et al. ${ }^{6}$ studied 18 patients with congestive heart failure. They administered $150 \mathrm{mg}$ of flosequinan and noted an increase of approximately $15 \%$ in $\mathrm{dP} / \mathrm{dt}_{\text {max }}$. They also noted an upward and leftward displacement of a single pressure-volume loop in several of their patients after flosequinan administration, which also suggested an enhancement in inotropy. Thus these data suggest that at doses of $\geq 100 \mathrm{mg}$ of flosequinan there is a modest inotropic effect, although not all data concurs.

Our investigation was undertaken to establish whether flosequinan had effects on left ventricular systolic and diastolic performance in addition to its systemic vasodilating action in a well-defined patient population with dilated congestive cardiomyopathy when flosequinan was administered in lower doses to produce modest hemodynamic effects. The data from this investigation indicate that when used with a standard therapeutic background, flosequinan had significant effects on systemic venous capacitance, arterial resistance, and left ventricular diastolic function at an average dose of $84 \mathrm{mg}$. In contrast, at these lower doses, flosequinan had no effect on left ventricular contractile function. By examining Fig. 3, two patients had a marked increase in their $\mathrm{E}_{\max }$ values, and the remaining eight patients had little or no change in their $\mathrm{E}_{\max }$ values. The two patients with enhanced inotropy both received $>100 \mathrm{mg}$ of flosequinan. This suggests either that there may be differences in individual patient responses to flosequinan because these two patients with enhanced inotropy had more mild contractile impairment or there is a dose-response effect of inotropy to flosequinan.

The observation that left ventricular active and passive relaxation were improved with flosequinan was unexpected. Recently it has been shown that, in patients with congestive heart failure and impaired left ventricular chamber elastance, left ventricular isovolumic relaxation has a wide range of impairment and therefore may be more sensitive to therapeutic intervention in these kinds of patients than left ven- tricular contractile function. ${ }^{30}$ There are several possible explanations for this effect of flosequinan on left ventricular diastolic function. Coronary blood flow, although normal at rest, may be reduced per unit mass in patients with congestive heart failure, making the subendocardium more susceptible to myocardial ischemia. ${ }^{31,32}$ Myocardial ischemia can produce delayed and incomplete relaxation, which can lead to impaired chamber distensibility. ${ }^{33}$ Flosequinan may have had a beneficial effect on these hemodynamic processes through a coronary vasodilating action. However, recent data from Cavero et al. ${ }^{34}$ in patients with congestive heart failure demonstrated that flosequinan in oral doses of $100 \mathrm{mg}$ did not change myocardial oxygen consumption, alter coronary sinus blood flow, or affect lactate production, suggesting that this is an unlikely possibility for the improvement in diastolic function.

Alternatively, by decreasing left ventricular size and by enhancing left ventricle pump function, flosequinan may have reduced plasma norepinephrine and transmyocardial norepinephrine flux, upregulating $\beta$-receptors, improving receptor-effector coupling to $\mathrm{G}$ proteins and cyclic adenosine monophosphate production and thus stimulating more efficient intracellular calcium handling. Alternatively, the direct effects of flosequinan on intracellular calcium handling may have improved isovolumic relaxation. An explanation for the improvement in left ventricular chamber compliance may be a reduction of extrinsic restraint or ventricular interaction. This would seem most likely and would be consistent with the effect observed in this homogeneous group of patients with dilated congestive cardiomyopathy. Carroll et al. ${ }^{35}$ showed that, in contrast to the effects of inotropic agents on chamber compliance, vasodilators cause a downward displacement of the diastolic pressure-volume curve consistent with relief of pericardial restraint or reduction in ventricular interaction. Further, a recent observation by Eichhorn et al. ${ }^{36}$ suggests that the explanation for changes in left ventricular end-diastolic pressure seen in patients like ours with congestive heart failure may be the result of both changes in isovolumic relaxation and chamber compliance. Whichever of these mechanisms are operative, flosequinan was shown in this investigation to have a beneficial effect on both of these measures of left ventricular diastolic function.

The observation has been made that most patients with congestive heart failure treated with flosequinan have an improvement in exercise tolerance when compared to placebo. ${ }^{5}$ For example, in a double-blind placebo controlled study to establish the 
effects of flosequinan on exercise tolerance, ${ }^{37}$ symptomatic status and exercise time improved significantly when compared to placebo ( $p<0.05$ for both). Moreover, exercise capacity is improved with flosequinan in patients with congestive heart failure without activation of the neurohumoral system. ${ }^{38}$ These beneficial effects may be related in part to the improvement in left ventricular diastolic dysfunction that was evident with flosequinan in this investigation. Thus these data suggest that the addition of flosequinan in low doses might be valuable in patients with congestive heart failure on a conventional therapeutic regimen by improving both systemic hemodynamics and left ventricular diastolic function and thus symptomatic status and exercise tolerance.

In conclusion, flosequinan is a new therapeutic agent for the treatment of congestive heart failure. Flosequinan has a mechanism of action that probably differs from other therapeutic agents currently available. By inhibition of the activation of transduction proteins or phospholipase $\mathrm{C}$, substantial systemic venous and arterial vasodilation occurs. Flosequinan also has an unexpected beneficial effect on left ventricular diastolic function that may be related to improved isovolumic relaxation or diminishment of restraint on the left ventricle; however, flosequinan has no significant effect at these lower doses on left ventricular contractility. This study emphasizes that new therapeutic agents like flosequinan may have a useful additive role to standard therapy in patients with congestive heart failure at lower doses where the potentially harmful effects of enhanced inotropy are not observed.

We thank Gary Viviano, BS, Henry Hawker, BS, Debbie Randall, LPN, Janet Petrusha, RN, Christina Frank, BS, Dan Montgomery, BS, and Kimberly Arment.

\section{REFERENCES}

1. Cowley A, Wynne R, Hampton J. The effects of BTS 49,465 on blood pressure and peripheral arteriolar and venous tone in normal volunteers. J Hypertens 1984;2:547-9.

2. Gristwood R, Beleta J, Bou J. Studies on the cardiac actions of flosequinan in vitro. Br J Pharmacol 1992;105:985-91.

3. Frodsham G, Jones R, Sim M. Effects of flosequinan: a novel arteriovenous dilating agent and its metabolite on bovine phosphodiesterase activity [Abstract]. $\mathrm{Br} \mathrm{J}$ Pharmacol 1989; 98:733.

4. Kessler P, Packer M. Hemodynamic effects of BTS 49,465 , a new long-acting systemic vasodilator drug, in patients with severe congestive heart failure. AM HEART J 1987;113:137-43.

5. Elborn J, Stanford C, Nicholls D. Effect of flosequinan on exercise capacity and symptoms in severe heart failure. Br Heart J 1989;61:331-5.

6. Burstein S, Semigran MJ, Dec GM, Boucher CA, Fifer MA. Positive inotropic and lusitropic effects of intravenous flosequinan in patients with heart failure. J Am Coll Cardiol $1992 ; 20: 822-9$.
7. Starling MR, Montgomery DG, Walsh RA. Load dependence of the single beat maximal pressure (stress)/volume ratios in humans. J Am Coll Cardiol 1989;14:345-53.

8. Starling MR, Dell' Italia LJ, Nusynowitz ML, Walsh RA, Little WC, Benedetto AR. Estimates of left-ventricular volumes by equilibrium radionuclide angiography: importance of attenuation correction. J Nucl Med 1984;25:14-20.

9. Starling MR, Gross MD, Walsh RA, Dell'Italia LJ, Montgomery DG, Squicciarini SA, Blumhardt R. Assessment of the radionuclide angiographic left ventricular maximum timevarying elastance calculation in man. J Nucl Med 1988;29:136881.

10. Glower DD, Spratt JA, Snow ND, Kabeg JS, Davis JW, Olson CO, Tyson CS, Sabiston DC, Rankin .JS. Linearity of the Frank-Starling relationship in the intact heart: the concept of preload recruitable stroke work. Circulation 1989;71:994-1009.

11. Sagawa K, Suga H, Shoukas A, Bakalar KM. End-systolic pressure-volume ratio: a new index of ventricular contractility. Am J Cardiol 1977;40:748-53.

12. Sagawa K. The ventricular pressure-volume diagram revisited. Circ Res 1978;43:677-87.

13. Sagawa $K$. The end-systolic pressure-volume relations of ventricle: definition, modifications and clinical use. Circulation 1981;63:1223-7.

14. Starling MR, Walsh RA, Dell' Italia LJ, Mancini GBJ, Lasher $\mathrm{JC}$, Lancaster JL. The relationship of various measures of end-systole to left ventricular maximum time-varying elastance in man. Circulation 1987;76:32-43.

15. Suga H, Hisano R, Goto Y, Yamada O. Normalization of endsystolic pressure-volume relation and $\mathrm{E}_{\max }$ of different sized hearts. Jpn Circ J 1984;48:136-43.

16. Berko B, Gaasch WH, Tanigawa N, Smith D, Craige E. Disparity between ejection and end-systolic indexes of left ventricular contractility in mitral regurgitation. Circulation 1987;75:1310-9.

17. Hsia $\mathrm{HH}$, Starling MR. Is standardization of left ventricular chamber elastance necessary? Circulation 1990;81:1826-36.

18. Weisfeldt ML, Scully HE, Fredericksen J. Hemodynamic determinants of maximum negative $\mathrm{dP} / \mathrm{dt}$ and periods of diastole. Am J Physiol 1974;227:H613-21.

19. Weiss JL, Frederiksen JW, Weisfeldt ML. Hemodynamic determinants of the time-course of fall in canine left ventricular pressure. J Clin Invest 1976;58:751-8.

20. Starling MR, Montgomery DG, Mancini GBJ, Walsh RA. Load independence of the rate of isovolumic relaxation in man. Circulation 1987;76:1274-81.

21. Mirsky I. Assessment of diastolic function: suggested methods and future considerations. Circulation 1983;69:836-41.

22. Slegowski MB, Miller C, Porter S. Simplified high-performance liquid chromatographic determination of flosequinan and its metabolite in plasma, serum and urine. J Chromatogr 1988;425:227-32.

23. Yates DB. Effects of flosequinan, a novel arteriovenous dilating agent, on isolated smooth muscle [Abstract]. Br J Pharmacol 1988; 94:431.

24. Yates DB, Holmes JR. Effect of flosequinan and other relaxants on rat aortic contractions stimulated by noradrenaline and phorbol ester [Abstract]. Br J Pharmacol 1988;95:819.

25. Yates DB, Hicks G, Sim MF. The effect of vasorelaxants which increase cyclic nucleotides on phosphoinositide metabolism [Abstract]. Br J Pharmacol 1989;96:112.

26. Fleming JW, Wisler PL, Watanabe AM. Signal transduction by $\mathrm{G}$ proteins in cardiac tissue. Circulation 1992;85:420-33.

27. Allcock AA, Frodsham G, Sim MR. Effects of flosequinan, a novel arteriovenous dilating agent, on cGMP levels in rat isolated aortic strips [Abstract]. Br J Pharmacol 1988;94:430.

28. Haas GJ, Binkley PF, Carpenter JA, Leier CV. Central and regional hemodynamics effects of flosequinan for congestive heart failure. Am J Cardiol 1989;63:1354-9.

29. Corin WJ, Monrad ES, Strom JA, Guistino S, Sonnenblick ES, 
LeJemtel T. Flosequinan: a vasodilator with positive inotropic activity. AM HEART J 1991;121:537-40.

30. Eichhorn EJ, Willard JE, Alvarez L, Kim AS, Glamann DB, Risser RC, Grayburn PA. Are contraction and relaxation coupled in patients with and without congestive heart failure? Circulation 1992;85:2132-9.

31. Magorien RD, Brown GP, Unverferth DV, Nelson S, Boudoulas H, Bambach D, Leier CV. Effects of hydralazine on coronary blood flow and myocardial energetics in congestive heart failure. Circulation 1982;65:528-32.

32. Vatner SF, Shannon R, Hittinger L. Reduced subendocardial coronary reserve: a potential mechanism for impaired diastolic function in the hypertrophied and failing heart. Circulation 1990;81:III-8-14.

33. Mirsky I. Assessment of passive elastic stiffness of cardiac muscle: mathematical concepts, physiologic and clinical considerations, directions of future research. Prog Cardiovasc Dis 1976;18:277-308

34. Cavero PG, DeMarco T, Kwasman M, Lau D, Liu M, Chatter- jee K. Flosequinan, a new vasodilator: systemic and coronary hemodynamics and neuroendocrine effects in congestive heart failure. J Am Col Cardiol 1992;20:1542-8.

35. Carroll JD, Lang RM, Neumann AL, Borow KM, Rajfer SL. The differential effects of positive inotropic and vasodilator therapy on diastolic properties in patients with congestive cardiomyopathy. Circulation 1986;74:815-25.

36. Eichhorn EJ, Hatfield B, Marcoux L. Determinants of enddiastolic pressure in patients with congestive heart failure. Circulation 1992;86(suppl I):379.

37. Packer M, Narahara KA, Elkayam U, Sullivan JM, Pearle DL, Massie BM, Creager MA. Double-blind, placebo-controlled study of the efficacy of flosequinan in patients with chronic heart failure. J Am Coll Cardiol 1993;22:65-72.

38. Schneeweiss A, Plich N, Green T, Wynne RD, Marmor A. Efficacy and safety of flosequinan, given over 3 days, evaluated by continuous hemodynamic monitoring. Cardiology 1989; $76: 201-5$.

\title{
Prognostic value of echocardiography in children with idiopathic dilated cardiomyopathy
}

\begin{abstract}
Mortality of pediatric patients with idiopathic dilated cardiomyopathy (IDC) is highest during the first 1 to 2 years the child is first seen. The echocardiograms of 72 infants and children with IDC were reviewed to determine whether left ventricular shortening fraction (LVSF), end-diastolic dimension, and wall mass were helpful in predicting outcome. Patients who were alive at last follow-up (group 1) were compared to those who died or required heart transplant (group 2). None of the indexes obtained at first examination were predictive of survival. Shortening fraction had improved significantly at follow-up 1 to 6 months after first examination in group 1 (15.1 \pm 0.9 to $24.4 \pm 1.8, p<0.001$ ) but remained unchanged in group 2 (14.1 \pm 1.5 to $15.3 \pm 3.2$ ). No significant trends were noted in left ventricular end-diastolic dimension or wall mass. The 1- and 5-year actuarial survival for all patients was $75 \%$ and $60 \%$, respectively. Persistent depression of LVSF $<15 \%$ was associated with a significantly lower survival rate (1-year $46 \%$; 5-year 29\%) compared to patients in whom follow-up LVSF was $>15 \%$ (1-year $97 \%$; 5 -year $90 \% ; p<0.05$ ). It is concluded that the follow-up LVSF obtained at least 1 month after first examination is helpful in identifying pediatric patients who would benefit from early cardiac transplantation. (AM HEART J 1994;128:133-6.)
\end{abstract}

Alan B. Lewis, MD Los Angeles, Calif.

From the Division of Cardiology, Childrens Hospital Los Angeles, Department of Pediatrics, University of Southern Califurnia.

Received for publication June 28, 1993; accepted Nov. 15, 1993.

Reprint requests: $\Lambda$ lan B. Lewis, MD, Division of Cardiology, Mail Stop 34, Childrens Hospital Los Angeles, PO Box 54700, Los Angeles, CA 900540700 .

Copyright 1994 by Mosby-Year Book, Inc $0002-8703 / 94 / \$ 3.00+0 \quad \mathbf{4} / \mathbf{1} / \mathbf{5 4 5 3 6}$
The clinical features and survival rates of infants and children with idiopathic dilated cardiomyopathy (IDC) have been well described..$^{1-3}$ Mortality is highest during the first 1 to 2 years after first examination. Survival rates at 1 year has been reported to be $75 \%$; at 5 years it has declined to $65 \% .{ }^{3}$ However, predictors of outcome have been variable and inconsistent. 